\title{
Influência da composição e das variáveis de processamento de formulações à base de argilas - uso em materiais refratários
}

\section{(Influence of composition and processing variables of clay-based formulations - use in refractory materials)}

\author{
J.M.R. Figueiredo, I. M. M. Fernandes, V. J. Silva, G. A. Neves, H. C. Ferreira, L. N. L. Santana \\ UAEMa, CCT, UFCG, R. Aprígio Veloso 882, Campina Grande, PB \\ jullymrc@gmail.com,ingridmayara_mf@hotmail.com,valmir_jspb@yahoo.com.br, \\ gelmires.neves@ufcg.edu.br,heber.ferreira@ufcg.edu.br,lisiane.navarro@ufcg.edu.br
}

\begin{abstract}
Resumo
As argilas são componentes fundamentais das massas para produção de materiais cerâmicos tradicionais, pois garantem plasticidade na etapa de conformação e resistência mecânica após a queima; também podem ser utilizadas nas formulações de cerâmicas refratárias devido ao desenvolvimento da fase mulita em temperaturas elevadas. O objetivo deste trabalho foi avaliar o efeito da composição, tempo de maturação e temperatura de queima em formulações à base de argilas para uso na fabricação de materiais refratários. As argilas foram caracterizadas por análise granulométrica, análise química, refratariedade, difração de raios X, análise térmica diferencial e termogravimétrica. As formulações contendo caulim mais ball clay e caulim mais esmectita foram submetidas à maturação por um período de 1 a 4 semanas. Em seguida, as massas foram conformadas por prensagem uniaxial obtendo-se corpos de prova, que foram submetidos a tratamento térmico em 1200, 1300 e $1400{ }^{\circ} \mathrm{C}$, e, posteriormente, analisados quanto às propriedades: absorção de água, retração linear de queima e resistência à flexão em três pontos. Os resultados obtidos evidenciaram que as formulações em estudo apresentaram após queima fases de quartzo, cristobalita e mulita como fase majoritária. A temperatura de queima foi o parâmetro que apresentou maior influência nas propriedades avaliadas, com maior efeito da temperatura mais alta $\left(1400{ }^{\circ} \mathrm{C}\right)$.
\end{abstract}

Palavras-chave: argilas, composição, refratário.

Abstract

Clays are key components of mass for traditional ceramic materials production because they ensure plasticity in the shaping step and mechanical resistance after firing. They can also be used in refractory ceramic formulations due to the development of mullite phase at high temperatures. The objective of this study was to evaluate the effect of composition, maturation time, and firing temperature on clay formulations for use in manufacturing refractory materials. The clays were characterized by particle size analysis, chemical analysis, refractoriness, $X$-ray diffraction, as well as differential thermal and thermogravimetric analyses. Formulations containing kaolin with ball clay and kaolin with smectite were submitted to a maturation period of 1 to 4 weeks. Next, the masses were shaped by uniaxial pressing to obtain test specimens, which were then subjected to heat treatment at 1200,1300 and $1400{ }^{\circ} \mathrm{C}$, and the following properties were subsequently analyzed: water absorption, linear firing shrinkage, and three-point flexural strength. The results showed that after firing the samples had quartz, cristobalite and mullite phases, with the latter as the major phase. The firing temperature was the parameter that had the greatest influence on the evaluated properties, with the highest temperature $\left(1400^{\circ} \mathrm{C}\right)$ exerting the most influence.

Keywords: clay, composition, refractory.

\section{INTRODUÇÃO}

A caulinita é composta principalmente por alumina e sílica, sendo esta última em maior proporção. Quando submetida ao processo de tratamento térmico sofre uma série de reações, nas quais estes óxidos se combinam favorecendo a nucleação e crescimento dos cristais de mulita, fase de grande influência nas propriedades mecânicas [1]. Nesse sentido alguns trabalhos [2,3] buscaram agregar valor aos caulins através de sua incorporação em formulações cerâmicas para a produção de porcelanas, corpos mulíticos e refratários [4]. Muitas pesquisas foram desenvolvidas utilizando o caulim como precursor principal em suas composições, entretanto, a caulinita é considerada como um argilomineral com baixa plasticidade em comparação com as ball clays e as esmectitas [5]. Em [6] destaca-se que é necessária a adição de pequenas quantidades de argila mais plásticas (entre 5 e 10\%) para conferir trabalhabilidade à massa e assegurar uma boa coesão em corpos cerâmicos a verde, além de permitir baixar a temperatura de sinterização, devido à presença de cátions alcalinos e alcalino-terrosos (agentes fundentes), como é o caso das argilas esmectíticas também denominadas de bentonitas.

As propriedades mecânicas dos produtos de materiais 
refratários são dependentes da composição das argilas e das condições de processamento empregados. Em [7] foi investigado o efeito da adição de $\mathrm{MgO}$ (até 4\% em massa) em três argilas de diferentes localidades sobre a morfologia da mulita desenvolvida bem como as propriedades físicas e mecânicas quando sinterizadas entre 1200 e $1400{ }^{\circ} \mathrm{C}$. Foi relatado que as principais fases mineralógicas após tratamento térmico foram mulita, quartzo e cristobalita; contudo, são necessários cerca de $4 \%$ de $\mathrm{MgO}$ para o desenvolvimento de cordierita. Ficou evidenciado através do MEV que a adição de $\mathrm{MgO}$ promoveu crescimento de grãos de mulita e a formação de cordierita pseudo-hexagonal [7]. Com relação ao tempo de maturação, alguns trabalhos na literatura tratam da influência desta variável na utilização de caulim. Em [8], verificou-se que o tempo de maturação influenciou no módulo de Young de uma mistura de caulim e cal, sendo avaliados os tempos de 0, 7, 14 e 28 dias. Em [9] foi avaliado o efeito do teor de umidade associado ao tempo de maturação da massa sobre as propriedades de placas cerâmicas laminadas. Os resultados evidenciaram que os teores de umidade associados ao tempo de maturação influenciaram nas propriedades das placas cerâmicas. Porém, neste trabalho foi utilizado tempo de maturação pequeno (4, 8 e 12 dias) e não foi realizada uma análise estatística. No presente trabalho foi avaliado um tempo de maturação de até quatro semanas e realizada uma análise estatística para verificar a significância desta variável nas propriedades físico-mecânicas.

Além da composição e do tempo de maturação, a etapa de conformação é fundamental, uma vez que durante este processo o material adquire a forma e características estruturais pretendidas, podendo ser conformado via plástica, seca ou líquida, sendo os métodos de conformação mais comuns: prensagem [10, 11], slip casting [12] e extrusão [13]; dentre estes, o processo de prensagem é bastante utilizado na cerâmica tradicional em virtude de sua elevada produtividade, fácil automatização e versatilidade, sendo possível a produção de peças de tamanhos e formas variadas. Além disso, permite a conformação de peças com baixa retração de secagem devido ao uso de baixos teores de umidade e, como consequência, apresentam estabilidade dimensional [14]. Um parâmetro de processamento que influencia fortemente as propriedades mecânicas é a temperatura de queima. Em [15] foram produzidos blocos porosos de mulita a partir de composições contendo caulim e resíduo de alumina utilizando tratamento térmico em forno convencional em temperaturas elevadas. Foi observado que os valores de absorção de água e porosidade aparente diminuíram com o aumento da temperatura, contribuindo para uma maior densificação e aumento na resistência mecânica, que foi relacionado também com as transformações de fases, destacando-se o aumento do teor de mulita, sendo esta fase responsável pela resistência mecânica dos materiais obtidos.

Um material cerâmico à base de mulita é um dos candidatos mais adequados para aplicações refratárias [16]. A refratariedade é a propriedade que o material tem de suportar altas temperaturas sem se deformar ou fundir. Dentre as diversas aplicações da mulita em materiais refratários, destacam-se: cadinhos, peças de isolamentos térmicos, revestimentos de fornos e reatores (para altas temperaturas), matriz e reforço de material compósito, componentes de motores de turbina, vedação elástica em altas temperaturas, catalisadores, membranas de filtração, acessórios para aeronaves espaciais e materiais leves, entre outras $[17,18]$. Diante desse contexto, este trabalho teve como objetivo avaliar o efeito da composição, tempo de maturação e da temperatura nas propriedades físico-mecânicas de peças cerâmicas obtidas a partir de formulações à base de argilas para uso na fabricação de materiais refratários mulíticos.

\section{MATERIAIS E MÉTODOS}

Para o desenvolvimento deste trabalho foram utilizados: caulim da empresa Rocha Minérios, localizada no município de Juazeirinho, PB, denominada de argila A; ball clay da indústria Armil Mineração do Nordeste, localizada em Parelhas, RN, sendo oriunda do município de Oeiras, PI, denominada de argila B; bentonita, argila bentonítica natural, policatiônica, proveniente do município de Cubati, PB, denominada de argila C. A caracterização foi efetuada utilizando: análise granulométrica por difração de laser (AG) em um granulômetro Cilas, 1064 LD; análise química por fluorescência de raios X em equipamento EDX720 Shimadzu; difração de raios X (DRX) com difratômetro XRD6000 Shimadzu, com radiação $\mathrm{CuK} \alpha(40 \mathrm{kV}, 30 \mathrm{~mA})$ e velocidade do goniômetro de $2 \%$ min e passo de $0,02^{\circ}$, com varredura de $5^{\circ}$ a $60^{\circ}$; análise térmica diferencial (ATD, RB-300, BP Engenharia), com taxa de aquecimento de $12,5{ }^{\circ} \mathrm{C} /$ min e temperatura máxima de $1000{ }^{\circ} \mathrm{C}$; e análise termogravimétrica (TG, sistema de análises térmicas TA $60 \mathrm{H}$ Shimadzu). Para todas as análises, as amostras apresentaram granulometria inferior à peneira $\mathrm{ABNT} \mathrm{n}^{\circ} 200(0,074 \mathrm{~mm})$. $\mathrm{O}$ valor aproximado do ponto de fusão incongruente das amostras foi calculado a partir da composição química por EDX utilizando a fórmula de Schuen [19], sendo:

Temperatura de fusão $=\frac{360+\mathrm{Al}_{2} \mathrm{O}_{3}-\mathrm{RO}}{0,228}\left(\mathrm{em}^{\circ} \mathrm{C}\right)(\mathrm{A})$

onde, $\mathrm{Al}_{2} \mathrm{O}_{3}$ é dada pela razão entre o teor de alumina e a soma de teores de alumina e sílica, e RO é a soma dos teores dos outros óxidos (em base calcinada, desconsiderando a fração da perda ao fogo). O valor obtido é aproximadamente igual à refratariedade ou cone pirométrico Orton equivalente da argila, que é definida pela norma ABNT NBR ISO 528 [20]. O valor de cone pirométrico é adimensional e cada temperatura de fusão corresponde a um cone [19].

Após a etapa de caracterização, foram formuladas duas composições a partir das matérias-primas, procurando-se estabelecer uma proporção de argilas que favorecesse a trabalhabilidade das massas, conforme Tabela I. Em seguida, as massas cerâmicas com teores de umidade adequados para o processo de conformação por prensagem (7\% segundo norma ABNT NBR 6457 [21]) ficaram em maturação pelos 
Tabela I - Composição das massas cerâmicas utilizadas. [Table I - Composition of the ceramic masses used.]

\begin{tabular}{cccc}
\hline Formulação & Argila A & Argila B & Argila C \\
\hline F1 & $90 \%$ & $10 \%$ & - \\
F2 & $90 \%$ & - & $10 \%$ \\
\hline
\end{tabular}

períodos de 1,2, 3 e 4 semanas, com o objetivo de melhorar a homogeneização da umidade na massa. Os corpos de prova foram conformados pelo processo de prensagem com pressão aplicada de 20,0 MPa por 20 s. Após esta etapa, os corpos de prova foram secos em estufa a $110{ }^{\circ} \mathrm{C}$ por $24 \mathrm{~h} \mathrm{e}$, em seguida, submetidos ao processo de queima nas temperaturas de 1200,1300 e $1400{ }^{\circ} \mathrm{C}$, com taxa de aquecimento de $5{ }^{\circ} \mathrm{C} / \mathrm{min}$ e com permanência de $60 \mathrm{~min}$ na temperatura máxima de queima.

Após a etapa de queima, os corpos de prova foram submetidos aos ensaios físico-mecânicos, determinandose: absorção de água (segundo o princípio de Arquimedes), retração linear de queima e resistência à flexão em três pontos. Para cada ensaio físico-mecânico realizado, foi feita a análise estatística utilizando-se um delineamento inteiramente casualizado em esquema fatorial $4 \times 3$ (tempo de maturação: 1,2,3 e 4 semanas; e temperatura: 1200, 1300 e $1400{ }^{\circ} \mathrm{C}$ ) e dez repetições usando o software estatístico Assistat 7.7 [22]. Para a comparação entre médias foi aplicado o teste de Tukey ao nível de 5\% de probabilidade. As fases formadas e a microestrutura desenvolvida após a queima também foram investigadas por meio de: difração de raios $\mathrm{X}(\mathrm{DRX})$, microscopia eletrônica de varredura (MEV) e microscopia eletrônica de transmissão (MET). Para o MEV e MET, foi realizado o tratamento com ácido fluorídrico (HF) a $40 \%$ por $10 \mathrm{~min}$, a fim remover a fase vítrea, para posterior análise da morfologia dos cristais de mulita. A caracterização morfológica por MEV foi realizada utilizando um microscópio FEI Quanta 200 FEG, com tensão de $20 \mathrm{kV}$ e ampliação de $60000 x$, e por MET em um microscópio FEI Morgagni 268D, com tensão de 50 kV e ampliação de até 180000x. Esta técnica foi empregada com o intuito de investigar a morfologia e os tamanhos de partículas das amostras. Os diâmetros das agulhas de mulita foram mensurados através da utilização do programa ImageJ a partir das imagens de obtidas por MET.

\section{RESULTADOS E DISCUSSÃO}

\section{Caracterização das matérias-primas}

Na Tabela II estão relacionados os valores das frações acumuladas abaixo de $2 \mu \mathrm{m}$, entre 2 e $20 \mu \mathrm{m}$ e superior a $20 \mu \mathrm{m}$, assim como os valores do diâmetro médio das partículas. A argila A apresentou maior diâmetro médio de partículas $(5,58 \mu \mathrm{m})$ e a argila $\mathrm{C}$ o menor diâmetro de $2,30 \mu \mathrm{m}$. Também foi possível observar teor elevado $(63,16 \%)$ de partículas abaixo de $2 \mu \mathrm{m}$ (comumente denominado fração argila) para a argila $\mathrm{C}$ e menor para argila B $(30,00 \%)$. No que se refere à faixa de diâmetro
Tabela II - Frações granulométricas (\%) por tamanho de partículas das matérias-primas.

[Table II - Grain size fractions (\%) by particle sizes of raw materials.]

\begin{tabular}{ccccc}
\hline Amostra & $\leq 2 \mu \mathrm{m}$ & 2 a $20 \mu \mathrm{m}$ & $>20 \mu \mathrm{m}$ & $\begin{array}{c}\text { Diâmetro } \\
\text { médio }(\mu \mathrm{m})\end{array}$ \\
\hline Argila A & 36,40 & 58,38 & 5,22 & 5,58 \\
Argila B & 30,00 & 70,00 & 0,00 & 4,59 \\
Argila C & 63,16 & 36,84 & 0,00 & 2,30 \\
\hline
\end{tabular}

de partículas entre 2 e $20 \mu \mathrm{m}$, a argila B apresentou maior teor $(70,00 \%)$ e a argila $\mathrm{C}$ a menor fração $(36,84 \%)$. Em [23] afirma-se que pós finos apresentam forte tendência à aglomeração, com isso na faixa entre 2 e $20 \mu \mathrm{m}$ pode conter parte argilosa, ou seja, frações grosseiras podem conter aglomerados da própria argila. A distribuição granulométrica tem grande influência no empacotamento de partículas a verde e na sinterização do produto queimado. Comparando os resultados da distribuição do tamanho de partículas da argila C com argilas bentoníticas de Boa Vista-PB [24], Pedra Lavrada-PB [25] e Sossego-PB [26], verificou-se que a argila em estudo apresentou maior quantidade de partículas finas. Conforme [27], quanto menor o tamanho de partícula, maior é a área superficial, sendo esse um fator importante para o aumento da reatividade entre as partículas, acelerando a cinética das reações e favorecendo o processo de difusão que dita as transformações de fase.

Os dados da composição química das matériasprimas estão relacionados na Tabela III. Os principais óxidos foram $\mathrm{SiO}_{2}, \mathrm{Al}_{2} \mathrm{O}_{3}, \mathrm{Fe}_{2} \mathrm{O}_{3}, \mathrm{~K}_{2} \mathrm{O}$ e $\mathrm{MgO}$, ao passo que $\mathrm{CaO}$ e $\mathrm{TiO}_{2}$ estavam presentes apenas em pequenas quantidades. Verificou-se que a quantidade de $\mathrm{SiO}_{2}$ (média de 49,0\%) indicou a presença de argilominerais, tais como caulinita e mica, bem como a presença de quartzo livre na amostra. A quantidade de $\mathrm{Al}_{2} \mathrm{O}_{3}$ foi relacionada à caulinita, apresentando elevados teores (média de 32,0\%) que tende a aumentar a refratariedade da massa argilosa. A razão das frações de alumina e sílica $\left(\mathrm{Al}_{2} \mathrm{O}_{3} / \mathrm{SiO}_{2}\right)$ variou de 0,50 para a argila $\mathrm{B}$ e 0,86 para a argila $\mathrm{A}$, cujos resultados foram de acordo com os obtidos na literatura [6, 28]. Quanto aos óxidos alcalinos $\left(\mathrm{K}_{2} \mathrm{O}\right.$ e $\left.\mathrm{Na}_{2} \mathrm{O}\right)$ e alcalinos terrosos $(\mathrm{MgO}$ e $\mathrm{CaO})$, observou-se que a argila $\mathrm{B}$ apresentou uma quantidade mais elevada de $\mathrm{K}_{2} \mathrm{O}(3,97 \%)$ e a argila $\mathrm{C}$ de $\mathrm{MgO}(2,45 \%)$ [29]. A principal função dos álcalis presentes nas amostras é atuar como fundentes, contribuindo para formação de fase vítrea e diminuir a sua refratariedade. Observou-se que a argila A apresentou baixo teor de óxido de ferro $\left(\mathrm{Fe}_{2} \mathrm{O}_{3}\right), 0,46 \%$, e teores mais elevados de $2,58 \%$ para a argila B e 6,40\% para a argila C. Logo, os produtos de queima destas argilas, provavelmente, apresentam cores claras, devido ao baixo teor de $\mathrm{Fe}_{2} \mathrm{O}_{3}$ [30]. Os valores de perda ao rubro (PR) variaram entre 7,77 e 13,27\% para as argilas B e A, respectivamente, que representaram a perda de água livre, adsorvida, coordenada e perda das hidroxilas dos argilominerais. Os resultados evidenciaram que as argilas possuíram composição química típica de argilas de 
Tabela III - Composição química (\% em massa) das matérias-primas.

[Table III - Chemical composition (wt\%) of the raw materials.]

\begin{tabular}{cccccccccc}
\hline Amostra & $\mathrm{SiO}_{2}$ & $\mathrm{Al}_{2} \mathrm{O}_{3}$ & $\mathrm{Fe}_{2} \mathrm{O}_{3}$ & $\mathrm{~K}_{2} \mathrm{O}$ & $\mathrm{MgO}$ & $\mathrm{CaO}$ & $\mathrm{TiO}_{2}$ & Outros & $\mathrm{PR}$ \\
\hline Argila A & 45,69 & 39,47 & 0,46 & 0,89 & nd & nd & nd & 0,22 & 13,27 \\
Argila B & 54,48 & 27,46 & 2,58 & 3,97 & 1,49 & 0,76 & 0,87 & 0,62 & 7,77 \\
Argila C & 46,76 & 29,13 & 6,40 & 0,52 & 2,45 & 0,80 & 1,15 & 1,63 & 11,16 \\
\hline nd - não detectado; $P R$ - perda ao rubro.
\end{tabular}

cerâmica técnica, e valores similares foram observados em outros estudos [2, 29]. Além disso, a argila B apresentou composição química similar às argilas plásticas do tipo ball clay do município de Oeiras no Piauí [19], sendo diferente das ball clays clássicas, visto que o teor de $\mathrm{K}_{2} \mathrm{O}$ foi elevado.

$\mathrm{Na}$ Fig. 1 estão apresentados os difratogramas de raios $\mathrm{X}$ das argilas A, B e C. Observou-se nas amostras das argilas A e B a presença predominantemente de caulinita (JCPDS 782110) e, em menor quantidade, por mica (JCPDS 83-1808) e

a)

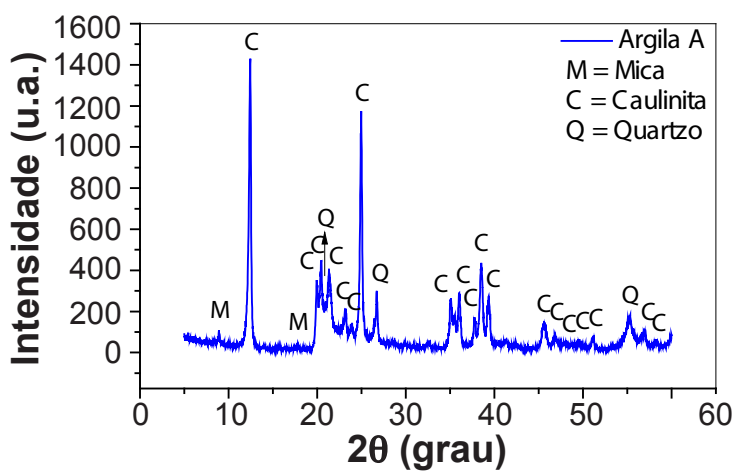

b)

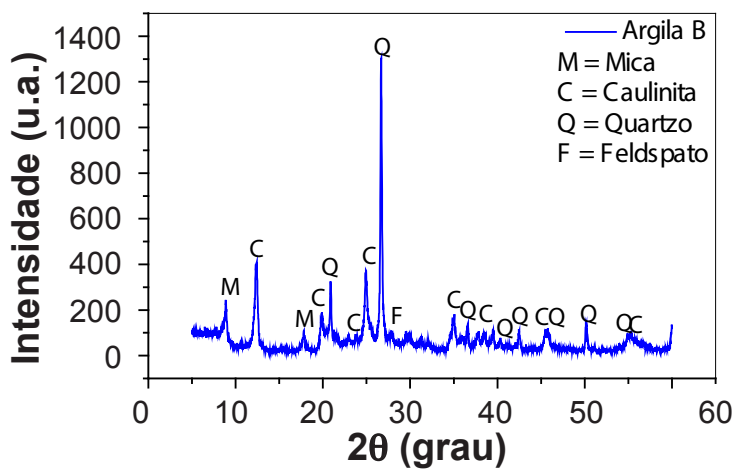

c)

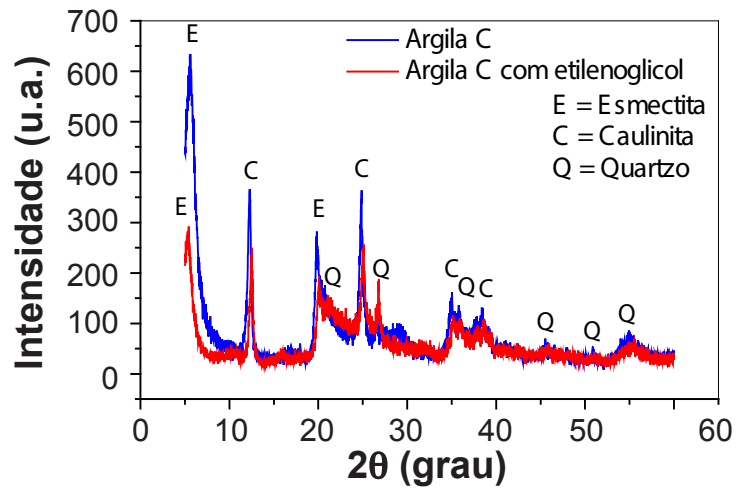

Figura 1: Difratogramas de raios $\mathrm{X}$ das argilas.

[Figure 1: X-ray diffraction patterns of the clays.] quartzo (JCPDS 46-1045) como fases cristalinas. A argila $\mathrm{B}$ apresentou ainda picos característicos de feldspato (JCPDS 89-8575). Por outro lado, a argila C apresentou picos de esmectita (JCPDS 13-0135), caulinita (JCPDS 79-1570) e quartzo (JCPDS 46-1045). Pôde-se verificar que
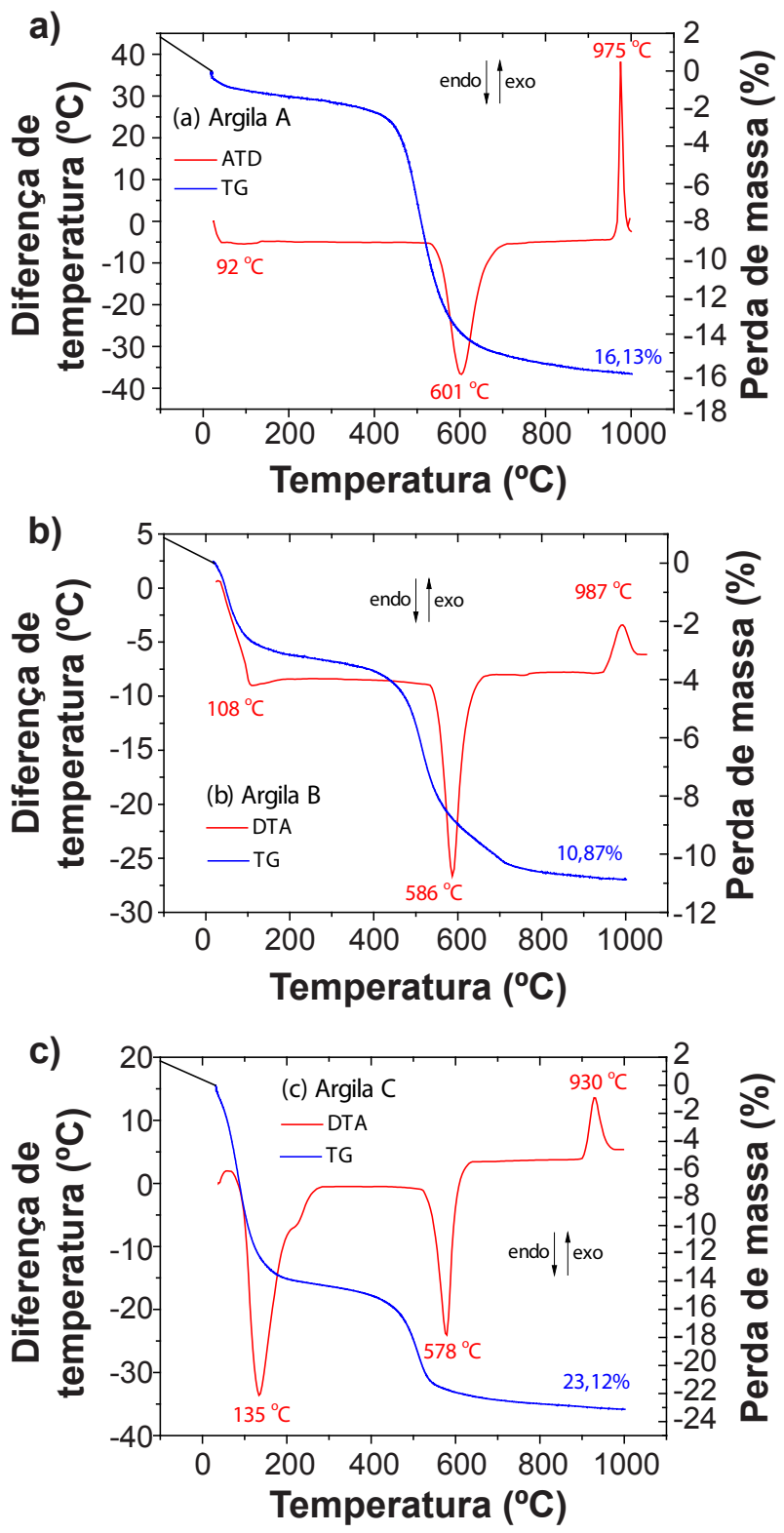

Figura 2: Curvas de análise térmica diferencial e termogravimétrica das argilas.

[Figure 2: Differential thermal analysis and thermogravimetry curves of the clays.] 
o $\mathrm{Fe}_{2} \mathrm{O}_{3}$ presente na argila $\mathrm{C}$ (Tabela III) estava relacionado ao ferro presente na estrutura cristalina do argilomineral do grupo da esmectita, já que não foi observada a presença de goetita, ilita ou outras fases que poderiam ser fontes de óxidos e hidróxidos de ferro. O deslocamento do pico referente ao argilomineral esmectítico foi confirmado após saturação com etilenoglicol, com o aumento da distância interplanar basal de 15 para $17 \AA$. Os picos característicos dos argilominerais encontrados nas argilas A, B e C foram compatíveis com outros trabalhos $[2,6,31]$.

Na Fig. 2 estão apresentadas, simultaneamente, as curvas das análises termodiferenciais (ATD) e termogravimétricas (TG) das amostras analisadas. Pela curva de ATD da argila A, pôde-se observar: um pequeno pico endotérmico com máximo em $92{ }^{\circ} \mathrm{C}$, caracterizando a presença de água livre; um intenso pico endotérmico a aproximadamente $601{ }^{\circ} \mathrm{C}$, que foi relacionado à presença de hidroxilas da folha octaédrica; e pico exotérmico com máximo em $975{ }^{\circ} \mathrm{C}$, característico da nucleação da mulita. De acordo com a curva de ATD da argila B, foram observadas as seguintes transformações térmicas: pico endotérmico com máximo em $108{ }^{\circ} \mathrm{C}$, característico da presença de água livre; um pico endotérmico a aproximadamente $586{ }^{\circ} \mathrm{C}$, característico da presença de hidroxilas da folha octaédrica; e pico exotérmico com máximo em $987{ }^{\circ} \mathrm{C}$, característico da nucleação da mulita. Analisando a curva de ATD da argila C, pôde-se observar: um grande pico endotérmico com máximo em $135{ }^{\circ} \mathrm{C}$, caracterizando presença de água livre e adsorvida; uma pequena banda endotérmica em torno de $150{ }^{\circ} \mathrm{C}$, caracterizando presença de água coordenada; pico endotérmico com máximo em $578{ }^{\circ} \mathrm{C}$ caracterizando presença de hidroxilas da folha octaédrica; e pico exotérmico com máximo em $930{ }^{\circ} \mathrm{C}$ característico da nucleação da mulita oriunda de quartzo- $\beta$ da estrutura amorfa previamente criada. Mediante análise das curvas de TG das argilas A, B e C, observou-se que a perda total de massa foi de $16,13,10,87$ e $23,12 \%$, respectivamente, correspondente às perdas de águas e hidroxilas dos argilominerais. Transformações térmicas semelhantes também foram observadas em $[32,33]$.

\section{Caracterização das massas cerâmicas}

Na Tabela IV estão apresentados os resultados das análises granulométricas por difração a laser das massas cerâmicas formuladas. Verificou-se que as massas apresentaram distribuições granulométricas semelhantes; o diâmetro médio das partículas foi de 5,50 $\mu \mathrm{m}$ para a formulação F1 e de 5,27 $\mu \mathrm{m}$ para a formulação F2. Também foi possível observar um elevado percentual de volume acumulado com diâmetro médio abaixo de $2 \mu \mathrm{m}$, equivalente a $36,45 \%$ para a formulação $\mathrm{F} 1$, correspondente à fração argila, e de $37,20 \%$ para a formulação F2. No que se refere ao volume acumulado de diâmetro de partículas entre 2 e $20 \mu \mathrm{m}$, para ambas as formulações foi em torno de $59 \%$.

Na Tabela V estão apresentados os dados da composição química das massas cerâmicas formuladas. Observou-se que a quantidade de óxido de silício $\left(\mathrm{SiO}_{2}\right)$ foi de $45,05 \%$ e 44,62\% para as formulações F1 e F2, respectivamente. No que diz respeito ao teor de $\mathrm{Al}_{2} \mathrm{O}_{3}$ pôde-se observar que a formulação $\mathrm{F} 2$ mostrou a maior percentagem, favorecendo a maior relação de $\mathrm{Al}_{2} \mathrm{O}_{3} / \mathrm{SiO}_{2}$ que foi de 0,86 ; para a formulação $\mathrm{F} 1$ essa relação foi de 0,84 . Observou-se pequeno teor de $\mathrm{Fe}_{2} \mathrm{O}_{3}$ nas massas que correspondeu a $0,62 \%$ para a formulação $\mathrm{F} 1$ e $0,78 \%$ para a formulação $\mathrm{F} 2$. Ambas as formulações apresentaram $\mathrm{K}_{2} \mathrm{O}$ e $\mathrm{MgO}$ na sua composição; estes podem atuar como fundente durante o processo de síntese, que favorece a formação da fase líquida, facilitando a difusão de íons de alumínio, beneficiando a nucleação e o crescimento da fase mulita [34, 35]. Verificou-se que as amostras apresentaram PR de 14,23 e $13,87 \%$ para as formulações F1 e F2, respectivamente, o que foi relacionada às perdas de águas e hidroxilas dos argilominerais.

A partir da composição química das massas cerâmicas formuladas (Tabela V) e da fórmula de Shuen foi calculado o ponto de fusão incongruente aproximado que está apresentado na Tabela VI; na mesma tabela também estão contidos os valores do cone pirométrico $(\mathrm{CO})$ equivalente ou refratariedade. Analisando os valores do cone pirométrico, observou-se que as formulações apresentaram

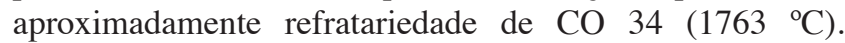
A refratariedade mínima, ou cone pirométrico mínimo, para que um material possa ser considerado refratário corresponde a CO $15\left(1435{ }^{\circ} \mathrm{C}\right)$. Logo, essas composições possuíram elevada refratariedade, sendo consideradas como

Tabela IV - Frações granulométricas (\%) por tamanho de partículas das formulações.

[Table IV - Grain size fractions (\%) by particle size of the formulations.]

\begin{tabular}{ccccc}
\hline Amostra & $\leq 2 \mu \mathrm{m}$ & 2 a $20 \mu \mathrm{m}$ & $>20 \mu \mathrm{m}$ & $\begin{array}{c}\text { Diâmetro } \\
\text { médio }(\mu \mathrm{m})\end{array}$ \\
\hline F1 & 36,45 & 59,34 & 4,21 & 5,50 \\
F2 & 37,20 & 59,68 & 3,12 & 5,27 \\
\hline
\end{tabular}

Tabela V - Composição química (\% em massa) das formulações. [Table V - Chemical composition (wt\%) of the formulations.]

\begin{tabular}{cccccccccc}
\hline Amostra & $\mathrm{SiO}_{2}$ & $\mathrm{Al}_{2} \mathrm{O}_{3}$ & $\mathrm{Fe}_{2} \mathrm{O}_{3}$ & $\mathrm{~K}_{2} \mathrm{O}$ & $\mathrm{MgO}$ & $\mathrm{CaO}$ & $\mathrm{TiO}_{2}$ & Outros & $\mathrm{PR}$ \\
\hline $\mathrm{F} 1$ & 45,05 & 37,85 & 0,62 & 1,11 & 0,82 & nd & 0,07 & 0,22 & 14,26 \\
$\mathrm{~F} 2$ & 44,62 & 38,52 & 0,78 & 0,70 & 1,14 & 0,10 & 0,05 & 0,22 & 13,87 \\
\hline
\end{tabular}

nd - não detectado; $P R$ - perda ao rubro. 
Tabela VI - Ponto de fusão incongruente aproximado e cone pirométrico equivalente das massas cerâmicas formuladas.

[Table VI - Approximate incongruent melting point and pyrometric cone equivalent of the formulated ceramic samples.]

\begin{tabular}{ccc}
\hline Amostra & $\begin{array}{c}\text { Ponto de fusão } \\
\text { incongruente }\left({ }^{\circ} \mathrm{C}\right)\end{array}$ & $\begin{array}{c}\text { Cone } \\
\text { pirométrico }\end{array}$ \\
\hline $\mathrm{F} 1$ & 1764 & 34 \\
$\mathrm{~F} 2$ & 1766 & 34 \\
\hline
\end{tabular}

silicoaluminosas refratárias.

\section{Caracterização das amostras tratadas termicamente}

Os difratogramas de raios $\mathrm{X}$ da evolução mineralógica das amostras referente às formulações F1 e F2, com tempo de maturação de uma semana, após tratamento térmico de 1200,1300 e $1400{ }^{\circ} \mathrm{C}$ estão apresentados na Fig. 3. Os resultados para os outros tempos de maturação não são apresentados, pois as amostras referentes às formulações F1 e F2 apresentaram comportamentos semelhantes. Observou-se para todas as amostras a presença das seguintes fases mineralógicas: mulita (JCPDS 79-1276), cristobalita (JCPDS 82-0512) e quartzo (JCPDS 46-1045), com predominância dos picos de mulita. Verificou-se também a presença de uma pequena banda, em $2 \theta$ variando de 15 a $30^{\circ}$, principalmente em $1200{ }^{\circ} \mathrm{C}$, característica da presença de material amorfo, ou seja, fase vítrea devido ao excesso de $\mathrm{SiO}_{2}$, como reportado na literatura $[15,35$, 36]. Nas formulações F1 e F2, pôde-se observar um pico de cristobalita caracterizado pela distância interplanar de 4,04 Å. Para a formulação F1, observou-se que houve intensificação desse pico com o aumento da temperatura de 1200 para $1300{ }^{\circ} \mathrm{C}$. Segundo [37], forma-se cristobalita devido ao excesso de sílica a partir de $1000{ }^{\circ} \mathrm{C}$. Em [1] explica-se em termos da cinética da reação que a mistura de alumina e sílica gera picos de cristobalita em amostras aquecidas por volta de $1200{ }^{\circ} \mathrm{C}$ devido à cristalização da sílica amorfa. Em $1400{ }^{\circ} \mathrm{C}$ observou-se redução na intensidade desses picos, indicando que ocorreu difusão na cristobalita, formando sílica amorfa; esta por sua vez reagiu com a alumina para formação da mulita $[1,38]$. A formulação $\mathrm{F} 2$, em 1200 e $1400{ }^{\circ} \mathrm{C}$, apresentou pico de cristobalita mais intenso quando comparado com a formulação F1. Fazendo uma correlação com a composição química, observou-se que a formulação F2 apresentou maior teor de óxidos fundentes; com o aumento da temperatura pode ter acelerado o processo de nucleação da cristobalita, provocando possivelmente o surgimento de uma grande quantidade de fase líquida de baixa viscosidade. Com o aumento da temperatura percebeu-se que os picos de mulita se intensificaram e os de quartzo diminuíram de intensidade, comportamento este observado nas duas formulações, o que pode estar relacionado com a maior interação entre alumina e sílica.
Na Fig. 4 estão apresentadas as micrografias obtidas por microscopia eletrônica de varredura (MEV) da formulação F1, com tempo de maturação de uma semana, após tratamento térmico a 1200 e $1400{ }^{\circ} \mathrm{C}$. Pôde-se observar que o tratamento a $1200{ }^{\circ} \mathrm{C}$ proporcionou uma microestrutura caracterizada pela formação de pequenas agulhas de mulita, provavelmente mulita primária, pois segundo alguns pesquisadores $[36,39$ 41] essa é a fase característica dessa faixa de temperatura quando argilas são submetidas a tratamentos térmicos. Com o aumento da temperatura para $1400^{\circ} \mathrm{C}$ observou-se a presença de cristais de mulita com maiores dimensões, que foi caracterizada pelo crescimento dos grãos anteriormente formados, tornando-os mais alongados, mantendo a forma do tipo agulhas, provavelmente de mulita secundária. A formação da mulita secundária ocorre da fase líquida transitória, onde acontece a precipitação dos cristais. Além disso, nas micrografias foi perceptível a presença de algumas partículas dispersas provavelmente de quartzo e também de fase vítrea. Não foi observada tendência de alinhamento preferencial, e sim uma estrutura com orientação planar e o entrelaçamento destas agulhas, que é típica do processo de prensagem, o que já era esperado, visto que essas micrografias representam os arranjos de grãos no plano perpendicular ao eixo da pressão aplicada durante a prensagem. Resultados similares foram obtidos em [42], obtendo-se uma textura planar perpendicular ao eixo da pressão aplicada. De acordo com [1], as impurezas quando em solução na estrutura de cristal de mulita reduzem a energia de superfície em algumas direções do cristal, conduzindo a um crescimento preferencial em outras direções, resultando na formação de grãos alongados. Portanto, quanto maior a solubilidade de impurezas na mulita mais acicular é o formato cristal. Em [43] ainda se relata que menores viscosidades da fase líquida aumentam a difusão das espécies envolvidas na cristalização da mulita e, consequentemente, atenuam o crescimento do
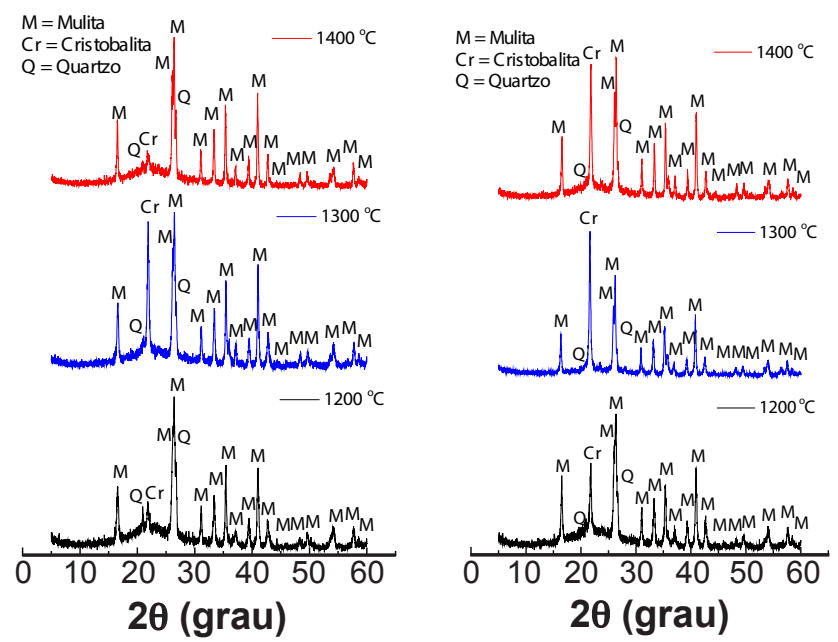

Figura 3: Difratogramas de raios $\mathrm{X}$ das amostras após tratamento térmico a 1200,1300 e $1400{ }^{\circ} \mathrm{C}$, com tempo de maturação de uma semana para as formulações F1 (a) e F2 (b).

[Figure 3: X-ray diffraction patterns of the samples after heat treatment at 1200,1300 and $1400^{\circ} \mathrm{C}$, with maturation of one week for formulations F1 (a) and F2 (b).] 

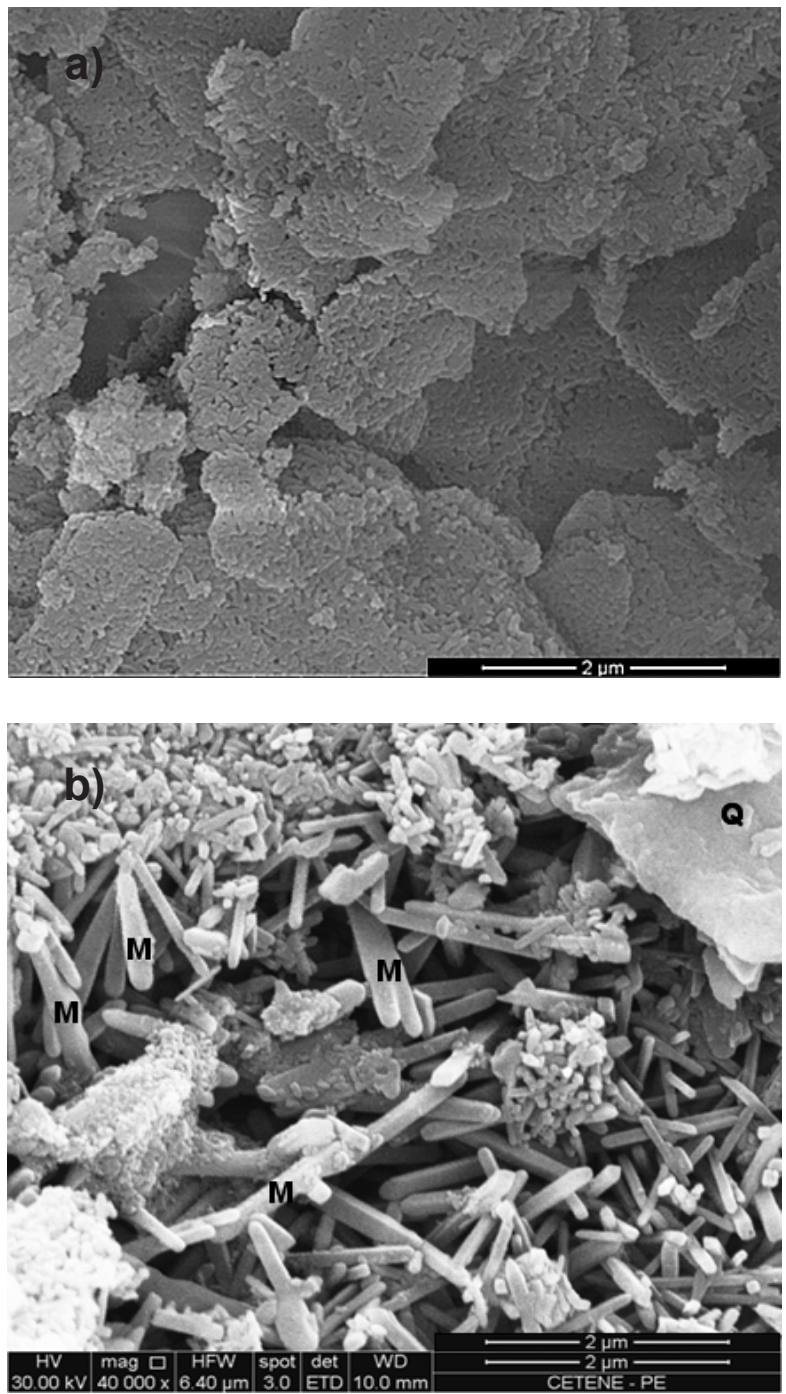

Figura 4: Micrografias obtidas por microscopia eletrônica de varredura da formulação $\mathrm{F} 1$, com tempo de maturação de uma semana, após tratamento térmico a $1200{ }^{\circ} \mathrm{C}$ (a) e $1400^{\circ} \mathrm{C}$ (b) $(\mathrm{M}$ mulita, Q - quartzo).

[Figure 4: SEM micrographs of F1 formulation with one week maturation and heat treated at $1200{ }^{\circ} \mathrm{C}\left(\right.$ a) and $1400{ }^{\circ} \mathrm{C}(\mathrm{b})(\mathrm{M}$ mullite, $Q$ - quartz).]

cristal. Em [44] acrescenta-se que o contínuo crescimento do tamanho dos grãos com o aumento da temperatura indica a possibilidade de transformação da mulita primária em mulita secundária.

Na Fig. 5 estão apresentadas as micrografias obtidas por microscopia eletrônica de transmissão (MET) da formulação $\mathrm{F} 1$, com tempo de maturação de uma semana, após tratamento térmico a 1200 e $1400{ }^{\circ} \mathrm{C}$. Observaram-se mulitas primária e secundária na forma acicular, crescendo com o aumento da temperatura resultando na formação de ligações fortes em estrutura com propriedades refratárias que melhoraram as propriedades mecânicas. Determinouse o comprimento (L) e diâmetro (D) médio das agulhas de mulita identificadas, obtendo-se o valor médio para a razão de aspecto $(\mathrm{RA}=\mathrm{L} / \mathrm{D})$, que estão apresentados na Tabela VII. As agulhas de mulita apresentaram uma estrutura
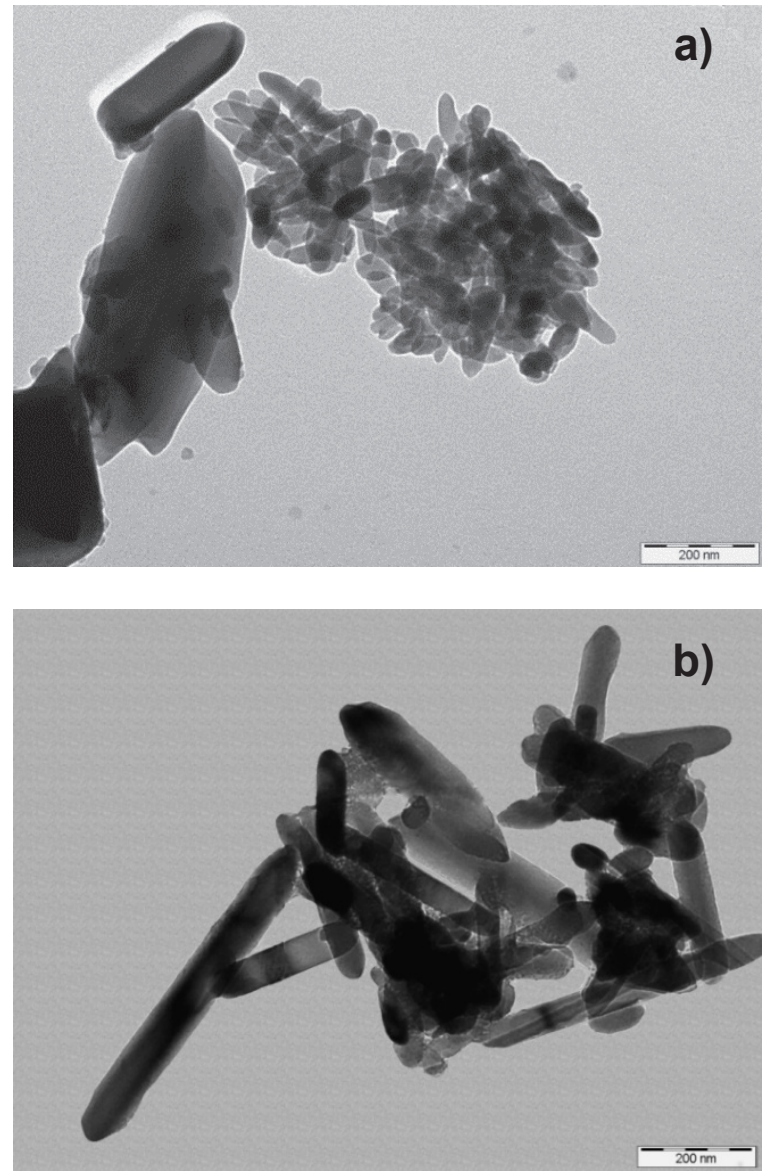

Figura 5: Micrografias obtidas por microscopia eletrônica de transmissão da formulação F1, com tempo de maturação de uma semana, após tratamento térmico a $1200{ }^{\circ} \mathrm{C}$ (a) e $1400{ }^{\circ} \mathrm{C}(\mathrm{b})$.

[Figure 5: TEM micrographs of $F 1$ formulation with one week maturation and heat treated at $1200^{\circ} \mathrm{C}($ a $)$ and $\left.1400^{\circ} \mathrm{C}(\mathrm{b}).\right]$

com orientação planar, observando-se seu entrelaçamento e cristais com tamanho variando de 0,14 a $0,35 \mu \mathrm{m}$. Para um material ser considerado nanométrico, no caso da mulita na forma de agulhas, o diâmetro dessas agulhas tem que ser menor ou igual a $100 \mathrm{~nm}$. De acordo com o exposto, considerou-se que o material em estudo foi nanométrico devido ao diâmetro das agulhas ser menor do que $100 \mathrm{~nm}$. As formas, dimensões e razões de aspecto estão alinhadas com as reportadas na literatura [45].

Na Tabela VIII estão contidos os valores médios de absorção de água em função da temperatura e do tempo de maturação para as formulações F1 e F2. Para a formulação F1, observou-se que a amostra da $2^{\mathrm{a}}$ semana apresentou maior absorção de água com temperatura de $1200{ }^{\circ} \mathrm{C}$, correspondente a $15,1 \%$. Para $1300{ }^{\circ} \mathrm{C}$, a amostra da $4^{\text {a }}$ semana apresentou menor absorção de água $(4,4 \%)$. Já em $1400{ }^{\circ} \mathrm{C}$, não houve influência estatisticamente significativa do tempo de maturação e os valores variaram de 0,09 a $0,20 \%$. Para a formulação F2, observou-se que as amostras da $2^{\text {a }}$ semana apresentaram menor absorção de água nas temperaturas de 1200 e $1300{ }^{\circ} \mathrm{C}$, correspondente a 12,9 e $6,8 \%$, respectivamente. Já para $1400{ }^{\circ} \mathrm{C}$, as amostras da $1^{\text {a }}$, $2^{\mathrm{a}} \mathrm{e} 4^{\mathrm{a}}$ semanas apresentaram menor absorção, não havendo 
Tabela VII - Dimensões das agulhas de mulita.

[Table VII - Dimensions of mullite needles.]

\begin{tabular}{cccccc}
\hline Amostra & $\begin{array}{c}\mathrm{L} \\
\text { médio } \\
(\mu \mathrm{m})\end{array}$ & $\begin{array}{c}\mathrm{D} \\
\text { médio } \\
(\mu \mathrm{m})\end{array}$ & $\begin{array}{c}\text { RA } \\
\text { mínimo }\end{array}$ & $\begin{array}{c}\text { RA } \\
\text { máximo }\end{array}$ & $\begin{array}{c}\text { RA } \\
\text { médio }\end{array}$ \\
\hline F1-1200 & 0,14 & 0,04 & 1,57 & 4,98 & 3,05 \\
F1-1400 & 0,35 & 0,07 & 3,44 & 8,09 & 5,22 \\
\hline
\end{tabular}

$L$ - largura; $D$ - diâmetro; $R A$ - razão de aspecto.

diferenças significativas. Verificou-se que a formulação $\mathrm{F} 2$, apesar de conter maior quantidade de óxidos fundentes $\left(\mathrm{Fe}_{2} \mathrm{O}_{3}, \mathrm{~K}_{2} \mathrm{O}, \mathrm{CaO}\right.$ e $\left.\mathrm{MgO}\right)$, apresentou maior absorção de água quando comparada com a formulação F1. Isto pode ter ocorrido devido à formulação $\mathrm{F} 1$ conter maior quantidade de $\mathrm{K}_{2} \mathrm{O}$ (óxido alcalino), que é um dos principais responsáveis pela formação de fase vítrea. Em temperaturas superiores a $1000{ }^{\circ} \mathrm{C}$ ocorreu uma reação deste óxido com o $\mathrm{SiO}_{2}$ proveniente da decomposição dos argilominerais formando fase líquida que promoveu, por ação de força de capilaridade, a densificação do material [46].Na Tabela IX estão contidos os valores médios de retração linear de queima em função da temperatura e tempo de maturação para as formulações F1 e F2. Para a formulação F1, observou-se em $1200{ }^{\circ} \mathrm{C}$

Tabela VIII - Valores médios de absorção de água (\%) em função da temperatura e tempo de maturação $\left(\mathrm{t}_{\mathrm{m}}\right)$.

[Table VIII - Average water absorption values (\%) according to temperature and maturation time $\left.\left(t_{m}\right).\right]$

\begin{tabular}{ccccc}
\hline \multirow{2}{*}{ Formulação } & $\mathrm{t}_{\mathrm{m}}$ & \multicolumn{3}{c}{ Temperatura de queima $\left({ }^{\circ} \mathrm{C}\right)$} \\
& 1 & $12,5 \mathrm{bA}$ & $6,3 \mathrm{aB}$ & $0,20 \mathrm{aC}$ \\
& 2 & $15,1 \mathrm{aA}$ & $6,5 \mathrm{aB}$ & $0,18 \mathrm{aC}$ \\
$\mathrm{F} 1$ & 3 & $11,8 \mathrm{cA}$ & $6,4 \mathrm{aB}$ & $0,09 \mathrm{aC}$ \\
& 4 & $12,6 \mathrm{bA}$ & $4,4 \mathrm{bB}$ & $0,09 \mathrm{aC}$ \\
\hline \multirow{3}{*}{$\mathrm{F} 2$} & 1 & $13,3 \mathrm{aA}$ & $7,5 \mathrm{aB}$ & $1,72 \mathrm{bC}$ \\
& 2 & $12,9 \mathrm{bA}$ & $6,8 \mathrm{bB}$ & $1,75 \mathrm{bC}$ \\
& 3 & $13,2 \mathrm{abA}$ & $7,4 \mathrm{aB}$ & $2,45 \mathrm{aC}$ \\
& 4 & $13,5 \mathrm{aA}$ & $7,4 \mathrm{aB}$ & $2,05 \mathrm{bC}$ \\
\hline
\end{tabular}

Obs: as médias seguidas da mesma letra minúscula nas colunas e maiúsculas nas linhas não diferem estatisticamente entre si ao nível de 5\% de probabilidade de acordo com o teste de Tukey. F1: coeficiente de variação $=5,2 \%$; média geral =6,3\%; desvio mínimo significativo para coluna $=0,38$; desvio mínimo significativo para linha $=0,35 . F 2$ : coeficiente de variação $=4,2 \%$; média geral =7,5\%; desvio mínimo significativo para coluna $=0,37$; desvio mínimo significativo para linha $=0,33$.

que a retração variou de 7,8 a $9,5 \%$. Em $1300{ }^{\circ} \mathrm{C}$ a variação foi de 12,4 a $12,6 \%$ para $1^{\text {a }}, 2^{a}$ e $3^{\text {a }}$ semanas; já a $4^{a}$ semana apresentou maior retração linear de queima correspondente a $13,6 \%$. Em $1400{ }^{\circ} \mathrm{C}$ a variação foi de 15,8 a $16,2 \%$, não sendo observadas diferenças significativas para esta temperatura. Para a formulação F2, observou-se que a amostra da $2^{\text {a }}$ semana apresentou maior retração linear de
Tabela IX - Valores médios de retração linear de queima (\%) em função da temperatura e tempo de maturação $\left(\mathrm{t}_{\mathrm{m}}\right)$.

[Table IX - Averages values of linear firing shrinkage (\%) according to temperature and maturation time $\left(t_{m}\right)$.]

\begin{tabular}{ccccc}
\hline \multirow{2}{*}{ Formulação } & $\mathrm{t}_{\mathrm{m}}$ & \multicolumn{4}{c}{ Temperatura de queima $\left({ }^{\circ} \mathrm{C}\right)$} \\
& (semana) & 1200 & 1300 & 1400 \\
\hline \multirow{3}{*}{$\mathrm{F} 1$} & 1 & $8,7 \mathrm{bC}$ & $12,4 \mathrm{bB}$ & $15,8 \mathrm{bA}$ \\
& 2 & $7,8 \mathrm{cC}$ & $12,5 \mathrm{bB}$ & $15,8 \mathrm{abA}$ \\
& 3 & $9,5 \mathrm{aC}$ & $12,6 \mathrm{bB}$ & $15,9 \mathrm{abA}$ \\
& 4 & $9,2 \mathrm{aC}$ & $13,6 \mathrm{aB}$ & $16,2 \mathrm{aA}$ \\
\hline \multirow{2}{*}{$\mathrm{F} 2$} & 1 & $8,8 \mathrm{bC}$ & $12,1 \mathrm{abB}$ & $14,9 \mathrm{abA}$ \\
& 2 & $9,0 \mathrm{aC}$ & $12,1 \mathrm{aB}$ & $14,9 \mathrm{abA}$ \\
& 3 & $8,8 \mathrm{bC}$ & $12,0 \mathrm{bB}$ & $14,8 \mathrm{bA}$ \\
& 4 & $8,9 \mathrm{bC}$ & $12,2 \mathrm{aB}$ & $14,9 \mathrm{aA}$ \\
\hline
\end{tabular}

Obs: as médias seguidas da mesma letra minúscula nas colunas $e$ maiúsculas nas linhas não diferem estatisticamente entre si ao nivel de 5\% de probabilidade de acordo com o teste de Tukey. F1: coeficiente de variação $=2,7 \%$; média geral $=12,5 \%$; desvio mínimo significativo para coluna $=0,39 ;$ desvio mínimo significativo para linha $=0,35 . F 2$ : coeficiente de variação $=0,83 \%$; média geral $=11,9 \%$; desvio mínimo significativo para coluna $=0,11$; desvio mínimo significativo para linha $=0,10$.

Tabela X - Valores médios de tensão de ruptura à flexão (MPa) em função da temperatura e tempo de maturação $\left(\mathrm{t}_{\mathrm{m}}\right)$. [Table $X$ - Average flexural breaking stress values (MPa) versus temperature and maturation time $\left(t_{m}\right)$.]

\begin{tabular}{ccccc}
\hline \multirow{2}{*}{ Formulação } & $\mathrm{t}_{\mathrm{m}}$ & \multicolumn{3}{c}{ Temperatura de queima $\left({ }^{\circ} \mathrm{C}\right)$} \\
& (semana) & 1200 & 1300 & 1400 \\
\hline \multirow{3}{*}{$\mathrm{F} 1$} & 1 & $36,8 \mathrm{abC}$ & $56,6 \mathrm{bcB}$ & $79,9 \mathrm{cA}$ \\
& 2 & $31,5 \mathrm{bC}$ & $52,7 \mathrm{cB}$ & $88,5 \mathrm{bcA}$ \\
& 3 & $43,1 \mathrm{aC}$ & $63,3 \mathrm{abB}$ & $97,1 \mathrm{abA}$ \\
& 4 & $41,9 \mathrm{aC}$ & $70,6 \mathrm{aB}$ & $101,2 \mathrm{aA}$ \\
\hline \multirow{3}{*}{$\mathrm{F} 2$} & 1 & $36,3 \mathrm{aC}$ & $61,5 \mathrm{aB}$ & $78,5 \mathrm{aA}$ \\
& 2 & $33,9 \mathrm{aC}$ & $57,2 \mathrm{abB}$ & $73,3 \mathrm{abA}$ \\
& 3 & $32,3 \mathrm{aC}$ & $54,8 \mathrm{bB}$ & $64,6 \mathrm{cA}$ \\
& 4 & $31,8 \mathrm{aC}$ & $57,2 \mathrm{abB}$ & $68,9 \mathrm{bcA}$ \\
\hline
\end{tabular}

Obs: as médias seguidas da mesma letra minúscula nas colunas e maiúsculas nas linhas não diferem estatisticamente entre si ao nível de 5\% de probabilidade de acordo com o teste de Tukey. F1: coeficiente de variação =12,5\%; média geral =63,6 MPa; desvio mínimo significativo para coluna =9,3; desvio mínimo significativo para linha =8,4. F2: coeficiente de variação $=8,9 \%$; média geral =54,2 $\mathrm{MPa}$; desvio mínimo significativo para coluna $=5,6$; desvio mínimo significativo para linha $=5,1$.

queima em $1200{ }^{\circ} \mathrm{C}(9,0 \%)$. Entretanto, em 1300 e $1400{ }^{\circ} \mathrm{C}$, não houve influência estatisticamente significativa do tempo de maturação. De forma geral, a formulação F1 apresentou maior média geral de retração $(12,5 \%)$ do que a formulação F2 $(11,9 \%)$

Na Tabela X estão contidos os valores médios de tensão de ruptura à flexão em função da temperatura e tempo de maturação para as formulações F1 e F2. Para a formulação $\mathrm{F} 1$, observou-se que em $1200{ }^{\circ} \mathrm{C}$ não houve diferenças 
estatisticamente significativas entre as amostras da $1^{\text {a }}, 3^{\text {a }}$ e $4^{\text {a }}$ semanas, com valores variando de 36,8 a $43,1 \mathrm{MPa}$, observando uma menor tensão de ruptura para a $2^{\mathrm{a}}$ semana (31,5 MPa). Já em 1300 e $1400{ }^{\circ} \mathrm{C}$ as amostras das $3^{\mathrm{a}}$ e $4^{\text {a }}$ semanas conduziram maior tensão de ruptura, havendo influência do tempo de maturação dos corpos de prova. Diante do exposto, observou-se que a melhor tensão de ruptura foi de 101,2 MPa alcançada na $4^{\mathrm{a}}$ semana, submetida a $1400{ }^{\circ} \mathrm{C}$. Este resultado pode estar relacionado com a cinética de reação, ou seja, uma maior temperatura favoreceu a formação de maior quantidade de mulita, cuja fase é responsável pela resistência mecânica de peças cerâmicas à base de argila, quando submetidas a elevadas temperaturas [34]. Entretanto, de acordo com a formulação F2, verificouse que não foram observadas diferenças significativas para as variações entre os valores de tempo de maturação para $1200{ }^{\circ} \mathrm{C}$. Para 1300 e $1400{ }^{\circ} \mathrm{C}$ as amostras da $1^{\mathrm{a}}$ semana apresentaram maior resistência mecânica, variando de 61,5 a $78,5 \mathrm{MPa}$, respectivamente. Assim como citado na literatura $[8,9]$, o tempo de maturação teve influência nas propriedades físico-mecânicas. Entretanto, não foi detectado um padrão na influência, sendo distintos os resultados quando associados às outras variáveis. Comparando os resultados das formulações F1 e F2, observou-se que a formulação F1 apresentou maior resistência mecânica, o que condiz com os resultados de absorção de água, pois esta foi a que apresentou menores valores, provavelmente por ter menor quantidade de poros, que são concentradores de tensão. Observando a Fig. 3, a formulação F1 apresentou picos de mulita com maior intensidade do que a formulação F2, provavelmente devido à maior quantidade formada. Segundo alguns autores $[1,44,47]$, esta fase é responsável pela resistência mecânica dos materiais obtidos a partir de argilas. Conforme também observado na Fig. 3, a formulação F2 apresentou picos de cristobalita com maiores intensidades, provavelmente esta fase atuou como inclusões, que podem comprometer as propriedades mecânicas [48].

\section{CONCLUSÕES}

As argilas A e B foram compostas predominantemente de caulinita e, em menor quantidade, por mica e quartzo; a argila B apresentou ainda o feldspato. Já a argila C apresentou esmectita, caulinita e quartzo. As formulações em estudo apresentaram após queima quartzo, cristobalita e mulita como fase majoritária. Em termos de propriedades físico-mecânicas, todos os fatores estudados (composição, tempo de maturação e temperatura de queima) conduziram influência significativa estatisticamente para as duas formulações (F1: $90 \%$ de argila A e 10\% de argila B; e F2: $90 \%$ de argila A e 10\% de argila C). No que se refere à composição, a formulação F1 apresentou maior resistência mecânica. Para o fator tempo de maturação não foi identificado um comportamento padrão entre os resultados dos ensaios para as duas formulações. Já o fator temperatura de queima foi o que gerou maior alteração nos resultados, com efeito mais significativo da temperatura mais alta (1400 $\left.{ }^{\circ} \mathrm{C}\right)$ para obtenção dos melhores resultados, além da elevada refratariedade. Portanto, diante dos resultados obtidos verificou-se que a composição química e granulometria das argilas, assim como as variáveis de processamento, influenciaram sobre as propriedades físico-mecânicas. Os valores de refratariedade e resistência mecânica sugerem o uso em algumas aplicações de produtos cerâmicos refratários, podendo-se destacar: tijolos refratários, revestimentos de fornos e cadinhos.

\section{AGRADECIMENTOS}

Os autores agradecemà CAPES e ao CNPq (309646/20138) pelo apoio financeiro. Nossos agradecimentos também ao CETENE (Centro de Tecnologias Estratégicas do Nordeste) pela realização da caracterização microestrutural.

\section{REFERÊNCIAS}

[1] M.V.M. Magliano, V.C. Pandolfelli, Cerâmica 56 (2010) 368.

[2] A.H. Aza, X. Turrillas, M.A. Rodriguez, T. Duran, P. Pen, J. Eur. Ceram. Soc. 34, 5 (2014) 1409.

[3] K. Boussois, N. Tessier-Doyen, P. Blanchart, J. Eur. Ceram. Soc. 34, 1 (2014) 119.

[4] Z. Zhou, G. Jin, H. Liu, J. Wu, J. Mei, Appl. Clay Sci. 97-98 (2014) 110.

[5] M.S. Zbik, Y.F. Song, R.L. Frost, J. Colloid Interface Sci. 349 (2010) 86.

[6] G. Lecomte-Nana, A. Mokrani, N. Tessier-Doyen, H. Boussois, H. Goure-Doubi, Ceram. Int. 39, 8 (2013) 9047.

[7] W.M.N. Nour, H.M. Awad, J. Aust. Ceram. Soc. 44 (2008) 27.

[8] F.G. Bell, Eng. Geology 42 (1996) 223.

[9] J.B. Azevedo, I.F. Leite, V.M. Sudério, G.A. Neves, H.L. Lira, L.N.L. Santana, Anais do $47^{\circ}$ Congr. Bras. Cerâm. (2003) 283.

[10] D. Kenfaui, D. Chateigner, M. Gomina, J.G. Noudem, J. Appl. Ceram. Technol. 8 (2011) 214.

[11] M. Romagnoli, M. Lassinantti Gualtieri, A. F. Gualtieri, R. Šliteris, R. Kažys, G. Tari, J. Eur. Ceram. Soc. 33 (2013) 2785 .

[12] K. Boussois, S. Deniel, N. Tessier-Doyen, D. Chateigner, C. Dublanche-Tixier, P. Blanchart, Ceram. Int. 39 (2013) 5327.

[13] K.J. Krakowiak, P.B. Lourenço, F.J. Ulm, J.Am. Ceram. Soc. 94 (2011) 3012.

[14] A. Custódio, M. Custódio, R.P. Coreggio, M. Cargnin, Rev. Técn. Cient. 3, 1 (2012) 215.

[15] V.J. Silva, M.F. Silva, W.P. Gonçalves, R.R. Menezes, G.A. Neves, H.L. Lira, L.N.L. Santana, Ceram. Int. 42, 14 (2016) 15471.

[16] E. Bartonickova, P. Ptacek, T. Opravil, F. Soukal, J. Masilko, R. Novotny, J. Svec, J. Havlica, Ceram. Int. 41, 10 (2015) 14116.

[17] J. Wu, H. Lin, J. Li, X. Zhan, J. Li, Adv. Eng. Mater. 12, 1-2 (2010) 71. 
[18] A.K. Singh, R. Sarkar, Ceram. Int. 42, 11 (2016) 12937. [19] P. Souza Santos, Ciência e tecnologia de argilas, $2^{\text {a }}$ Ed., Edgar Blücher, S. Paulo (1992).

[20] Assoc. Brasil. Normas Téc., NBR ISO 528, "Produtos refratários - determinação do cone pirométrico equivalente (refratariedade)", Rio de Janeiro (2014).

[21] Assoc. Brasil. Normas Téc., NBR 6457, “Amostras de solo - preparação para ensaios de compactação e ensaios de caracterização", Rio de Janeiro (2016).

[22] F.A. Silva, C.A.V. Azevedo, in World Congr. Comput. Agric., 7,Am. Soc.Agric. Biol. Eng., Reno-NV-USA (2009). [23] R. Oliveira, A.R. Studart, R. G.Pileggi, V.C. Pandolfelli, Dispersão e empacotamento de partículas, princípios $e$ aplicações em processamento cerâmico, Fazendo Arte Ed., S. Paulo (2000).

[24] J.M.R. Costa, I.A. Silva, H.S. Ferreira, R.R. Menezes, G.A. Neves, H.C. Ferreira, Cerâmica 58 (2012) 419.

[25] I.A. Silva, J.M.R. Costa, R. R. Menezes, H.S. Ferreira, G.A. Neves, H.C. Ferreira, Rev. Esc. Minas 66, 4 (2013) 485.

[26] I.D.S. Pereira, I.A. Silva, J.M. Cartaxo, R.R. Menezes, L.N.L. Santana, G.A. Neves, H.C. Ferreira, Cerâmica 60, 354 (2014) 223.

[27] C.Y. Chen, W.H. Tuan, Ceram. Int. 27, 7 (2001) 795.

[28] I.K. Oikonomopoulos, M. Perraki, N. Tougiannidis, T. Perraki, H.U. Kasper, M. Gurk, Appl. Clay Sci. 103 (2015) 1.

[29] S. Mahmoudi, A. Bennour, A. Meguebli, E. Srasra, F. Zargouni, Appl. Clay Sci. 127-128 (2016) 78.

[30] A.N. Nzeukou, N. Fagel, B.K.A. Njoya, V.R.E. Medjo, U.C. Melo, Appl. Clay Sci. 83-84 (2013) 238.

[31] R.R. Menezes, G.A. Neves, H.C. Ferreira, Rev. Eletr. Mater. Proc. 9, 3 (2014) 118.

[32] M. Issaoui, L. Limousy, B. Lebeau, J. Bouaziz, M. Fourati, C. R. Chim. 19 (2016) 496.
[33] N. Garg, J. Skibsted, Cem. Concr. Res. 79 (2016) 101. [34] L.N.L. Santana, J. Gomes, G.A. Neves, H.L. Lira, R.R. Menezes, A.M. Segadães, Appl. Clay Sci. 87 (2014) 28.

[35] W.P. Gonçalves, V.J. Silva, J. Gomes, R.R. Menezes, G.A. Neves, H.C. Ferreira, L.N.L. Santana, Cerâmica 60 (2014) 316.

[36] C.J. McConville, W.E. Lee, J. Am. Ceram. Soc. 88, 8 (2005) 2267.

[37] H. Cheng, L. Qinfu, J. Yang, S. Ma, R.L. Geada, Thermoch. Acta 545 (2012) 1.

[38] P.C. Yu, Y.W. Tsai, F.S. Yen, W.P. Yang, C.L. Huang, J. Eur. Ceram. Soc. 35 (2015) 673.

[39] S. Lee, Y.J. Kim, H. Moon, J. Am. Ceram. Soc. 82, 10 (1999) 2841.

[40] W.E.Lee, G.P.Souza,C.J.Mcconville, T.Tarvornpanich, Y. Iqbal, J. Eur. Ceram. Soc. 28, 2 (2008) 465.

[41] S. Deniel, N. Tessier-Doyen, C. Dublanche-Tixier, D. Chateigner, P. Blanchart, J. Eur. Ceram. Soc. 30 (2010) 2427. [42] F. Gridi-Bennadji, D. Chateigner, G. Di Vita, P. Blanchart, J. Eur. Ceram. Soc. 29 (2009) 2177.

[43] D.H. Piva, R.H. Piva, J. Venturini, M.R. Morelli, C.P. Bergmann, Cerâmica 61, 359 (2015) 374.

[44] Y.F. Chen, M.C. Wang, M.H. Hon, J. Eur. Ceram. Soc. 24 (2004) 2389.

[45] J. Cao, X. Dong, L. Li, Y. Dong, S. Hampshire, J. Eur. Ceram. Soc. 34 (2014) 3181.

[46] C.M.F. Vieira, S.N. Monteiro, J.D. Filho, Cerâm. Ind. 6, 6 (2001) 43 .

[47] L. Carbajal, F. Rubio-Marcos, M.A. Bengochea, J.F. Fernandez, J. Eur. Ceram. Soc. 27 (2007) 4065.

[48] J.J. Liang, Q.H. Lin, X. Zhang, T. Jin, Y.Z. Zhou, X.F. Sun, B.G. Choi, I.S. Kim, J.H. Do, C.Y. Jo, J. Mater. Sci. Techn. 33, 2 (2017) 204.

(Rec. 05/10/2016, Rev. 14/12/2016, 15/03/2017, Ac. 16/03/2017) 